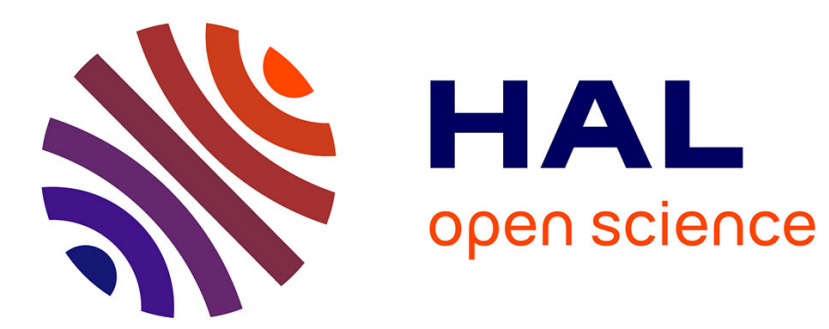

\title{
Adorno et la dialectique de la liberté
}

\author{
Isabelle Aubert
}

\section{To cite this version:}

Isabelle Aubert. Adorno et la dialectique de la liberté. Cahiers philosophiques, 2018, 154 (3), pp.79. 10.3917/caph1.154.0079 . hal-02948510

\section{HAL Id: hal-02948510 https://hal.science/hal-02948510}

Submitted on 24 Sep 2020

HAL is a multi-disciplinary open access archive for the deposit and dissemination of scientific research documents, whether they are published or not. The documents may come from teaching and research institutions in France or abroad, or from public or private research centers.
L'archive ouverte pluridisciplinaire HAL, est destinée au dépôt et à la diffusion de documents scientifiques de niveau recherche, publiés ou non, émanant des établissements d'enseignement et de recherche français ou étrangers, des laboratoires publics ou privés.

\section{다(1) $\$$}

Distributed under a Creative Commons Attribution - NonCommercial| 4.0 International 


\title{
Adorno et la dialectique de la liberté
}

\author{
Isabelle Aubert \\ (Université Paris 1 Panthéon-Sorbonne, ISJPS)
}

\begin{abstract}
Cet article s'intéresse au traitement que réserve Adorno à la philosophie morale de Kant dans la Dialectique négative et dans les cours magistraux qui ont préparé cet ouvrage, Probleme der Moralphilosophie et Zur Lehre von der Geschichte und von der Freiheit. Adorno entretient un rapport ambivalent à la théorie morale de Kant en la considérant à la fois comme étant grevée de « contradictions internes » et comme la « philosophie morale par excellence ». L'article prend pour fil directeur le reproche majeur qu'adresse Adorno à Kant d'avoir essayé de résoudre l'antinomie de la liberté et de négliger la dialectique de la liberté et de la non-liberté. Après avoir dégagé les déplacements sémantiques opérés par Adorno, il montre comment c'est en se situant par rapport à Kant qu'Adorno renouvelle la réflexion morale.
\end{abstract}

Contemporain d'Auschwitz, Theodor W. Adorno développe un rapport à la philosophie qui est à jamais imprégné des témoignages des camps de la mort ${ }^{1}$. Auschwitz incarne en effet la preuve irréfutable de «l'échec de la culture ${ }^{2}$ », et avec elle de la tradition philosophique. Dans divers écrits antérieurs, notamment dans la Dialectique de la raison, Adorno et Horkheimer ont expliqué cet échec de la raison qui, sans entreprendre de réflexion sur soi, régresse en devenant un instrument de domination. Vingt ans plus tard, dans la Dialectique négative, Adorno s'intéresse à ce qu'il reste de la philosophie ou à ce qui peut en être sauvé, sachant que toute «culture consécutive à Auschwitz (...) n'est qu'un tas d'ordure », en étant soit la même culture « coupable et minable » qui a fait naître la barbarie soit un refus de toute culture convergeant avec la barbarie, soit encore un silence complice ${ }^{3}$. L'autocritique du penser que développe la Dialectique négative prend pour objet d'investigation principal la métaphysique, considérée souvent comme "l'achèvement » de toute culture ${ }^{4}$, et cela en commentant et critiquant les approches de Heidegger, Hegel et Kant, et leurs vues connexes sur l'ontologie (Heidegger), la philosophie de l'histoire (Hegel), la théorie de la connaissance et la théorie morale (Kant), laquelle retiendra notre attention ici. Kant ayant démontré le primat de l'intérêt pratique de la raison sur son intérêt théorique et ce faisant, selon une interprétation acceptée aujourd'hui, le passage d'une métaphysique spéculative à une métaphysique pratique fondée sur l'Idée de libertés, l'enquête de la Dialectique négative intitulée "Liberté pour une métacritique de la raison pratique », qui porte sur la théorie morale kantienne, a également des répercussions sur les bases de cette métaphysique.

\footnotetext{
${ }^{1}$ On notera le parallèle avec les philosophes français écrivant dans les années quarante qui, de la même manière, redonneront des orientations nouvelles à la philosophie, tels Emmanuel Lévinas, Georges Canguilhem ou JeanPaul Sartre, ainsi qu'avec les membres du groupe «Socialisme ou Barbarie » fondé en 1948 (Claude Lefort, Cornelius Castoriadis, Jean-François Lyotard). Sur le parallèle entre Adorno et les philosophes français, voir M. Cohen-Halimi, Stridences spéculatives. Adorno, Lyotard, Derrida, Paris, Payot, 2014.

${ }^{2}$ T. W. Adorno, Dialectique négative [1966], trad. Collège de philosophie, Paris, Payot, 1978, p. 287.

${ }^{3}$ Ibid.

${ }^{4}$ Ainsi I. Kant, Critique de la raison pure [1781], trad. A. Renaut, Paris, GF, 2001, AK III 549, p. 685.

${ }^{5}$ I. Kant, Critique de la raison pratique [1788], trad. J.-P. Fussler, Paris, GF, 2003, AK V, 123, p. 247 et Métaphysique des mours, trad. A. Renaut, Paris, GF, 1994, Introduction, AK VI, 216-217, p. 166. Si le débat semble tranché aujourd'hui en faveur d'un « renouvellement pratique de la métaphysique chez Kant » (selon l'article au titre éponyme de M. Morais, Revue philosophique de Louvain, t. 103, n³, 2005, p. 301-330), les interprétations rivales (fin de la métaphysique versus naissance de la métaphysique chez Kant) s'opposaient lors de l'écriture de la Dialectique négative. Adorno ne pouvait ignorer les vues contraires des néo-kantiens et celles de Heidegger (dans Kant et le problème de la métaphysique) ainsi que des enjeux de ces lectures pour le statut de la philosophie pratique.
} 
Le fait que la Schoah ait pu avoir lieu dépasse à ce point le cadre de référence des théories morales antérieures à la Seconde Guerre mondiale que la question de l'obsolescence de la philosophie morale vient à se poser. Cette conclusion serait sans doute la plus logique au vu des événements passés. Et pourtant sonner le glas de la philosophie morale revient aussi par le même geste à se priver de catégories pour qualifier l'horreur d'Auschwitz. D'emblée, le problème posé par la «philosophie morale» est délicat; Adorno s'attache à montrer sa complexité sans la simplifier.

Le contexte d'une société répressive totale - qui fournit des conditions sociopsychologiques favorables pour insensibiliser progressivement toute une population à des actes de barbarie - invalide en bloc les théories morales précédentes. Aussi diverses qu'aient été celles-ci, qu'il s'agisse d'éthiques cognitivistes ou d'éthiques du sentiment moral, elles reposent toujours sur l'hypothèse selon laquelle la socialisation des membres d'une société donne lieu également à leur formation en tant que sujets moraux. En présupposant une socialisation non déformée, les éthiques ${ }^{6}$ antérieures à Auschwitz comportent toutes un impensé : elles ignorent les conditions historiques et sociales qui préexistent au jugement moral et qui nécessairement l'influencent.

La question morale revient de façon récurrente dans les écrits d'Adorno, comme en témoignent ses œuvres principales, Minima Moralia. Réflexion sur la vie mutilée, rédigée de 1944 à 1947, la Dialectique de la raison, coécrite avec Max Horkheimer, ou encore la Dialectique négative (1966). Les textes des années soixante, la Dialectique négative et ses cours préparatoires, Probleme der Moralphilosophie (1963) et Zur Lehre von der Geschichte und von der Freiheit (1964/1965), adoptent de façon systématique une perspective métaéthique pour étudier la morale ou plutôt sa possibilité même, et entreprennent, à cette occasion, une discussion serrée avec la philosophie morale kantienne. Nous allons privilégier leur étude dans cet article.

Ces textes d'Adorno sont à première lecture assez déconcertants car ils exposent par le menu une attitude ambivalente par rapport à la théorie morale du philosophe de Koenigsberg. D'un côté, la liste de critiques signalant les « contradictions internes ${ }^{7}$ » de l'éthique kantienne est longue. Leur grief principal est que Kant donne une assise à la morale bourgeoise qui a conduit aux atrocités du national-socialisme - point que signalait déjà la Dialectique de la raison en considérant que la Juliette libertine du marquis de Sade applique les méthodes kantiennes de la maîtrise de soi et ce faisant « incarne le plaisir de détruire la civilisation par ses propres armes $»^{8}$.

D'un autre côté, et par différence avec toute une série de critiques à commencer par celles de Hegel, Adorno ne conçoit pas une philosophie morale non kantienne. Il est ferme à ce sujet dans Probleme der Moralphilosophie : "Le fait est que nous pouvons dire que la philosophie morale de Kant est la philosophie morale par excellence, la philosophie morale en tant que telle ${ }^{9} »$. Les approches alternatives, les éthiques du bien entendues au sens large, qui mettent l'accent sur les valeurs sociales (Hegel) ou leur critique (Nietzsche), rateraient le problème moral fondamental découvert par Kant : la tension entre le particulier et l'universel, entre l'individu empirique et l'intérêt général ${ }^{10}$. L'aspect crucial réside dans cette interpellation de l'individu par une obligation inconditionnelle et l'opposition entre les deux tendances qui s'expriment alors qui met en évidence une non coïncidence, une tension entre

\footnotetext{
${ }^{6}$ À la suite d'Adorno dans ses cours de philosophie morale, j'utilise « éthique » en un sens synonyme de «théorie morale».

${ }^{7}$ T. W. Adorno, Probleme der Moralphilosophie [1963], Francfort, Suhrkamp, 1996, p. 242. [Les traductions de PM sont miennes.]

${ }^{8}$ T. W. Adorno, M. Horkheimer, Dialectique de la raison [1947], trad. E. Kaufholz, Paris, Gallimard, tel, 1974, 2011, p. 104. [Dorénavant noté $D R$.]

${ }^{9}$ T. W. Adorno, Probleme der Moralphilosophie, op. cit., p. 158.

${ }^{10}$ Ibid., p. 34.
} 
l'individu socialisé et son environnement social. Adorno poursuit cette idée selon laquelle l'expérience morale fait prendre conscience à l'individu de son impossible conciliation avec un universel. Cet aspect dote selon lui l'éthique kantienne d'une portée critique inédite : elle apporte « une image du possible, ou mieux une image du possible qui ne fait pas image ${ }^{11}$ ».

Enfin, Kant a mis en lumière ce qui est central pour Adorno, le fait qu'il y a une «dialectique de la moralité ${ }^{12}$ ». Ou encore que « le porteur de la raison, le sujet, [a] la possibilité d'être à la fois libre et non-libre ${ }^{13}$ » : la troisième antinomie de la Critique de la raison pure représente le principe même de la philosophie morale et son contenu de vérité tient précisément, aux yeux d'Adorno, dans l'existence de cette contradiction insoluble. Ce qui fait dire à la Dialectique négative : "La question de savoir si la volonté est libre ou non, nous force à un «ou bien, ou bien» tout aussi contraignant que problématique ${ }^{14}$.» La «contradiction [étant] dans la chose ${ }^{15}$ » et non dans la méthode, cette alternative est illusoire. En énonçant l'antinomie de la liberté, Kant a perçu le problème fondamental de la morale, mais son erreur a été de chercher à la résoudre en suivant « la logique de la noncontradiction ${ }^{16} »$. Il semblerait que le rapport complexe d'Adorno à Kant vienne en partie des tensions qu'il dégage au sein même de la théorie kantienne - la Dialectique de la raison ne constatait-elle pas déjà que les « concepts kantiens sont ambigus ${ }^{17}$ ? »

Les écrits adorniens oscillent donc entre des moments de critiques déconstructives et des moments de sauvetage de la théorie morale de Kant. La position d'Adorno se distingue par son rejet de deux approches réductrices et contraires de l'éthique kantienne : il refuse de réduire l'éthique kantienne à un simple rigorisme moral (qu'illustre l'attitude du nazi Eichmann déclarant avoir agi de façon kantienne) tout autant qu'il refuse de réhabiliter sans critique, à l'inverse de Hannah Arendt, cette philosophie pratique ${ }^{18}$.

Afin d'étudier la «métacritique de la raison pratique pure », menée dans la Dialectique négative et ses travaux préparatoires, nous prendrons pour fil directeur le reproche majeur qu'adresse Adorno à Kant d'avoir cherché une solution à la troisième antinomie. En suivant cette ligne d'analyse, nous montrerons comment le renouvellement de la réflexion morale effectué par Adorno à partir de la dialectique de la liberté ne prend sens qu'à la lumière de son opposition à la philosophie pratique de Kant tout autant que grâce à une interaction constante avec elle. Les résultats successifs d'une telle analyse vont nous permettre de défendre une thèse : la conception adornienne de la morale se trouve dans un enchevêtrement intime des pensées d'Adorno et de Kant. C'est de façon progressive que nous étayerons cette idée en nous penchant d'abord sur les problèmes perçus par Adorno dans la réduction de l'antinomie de la liberté (opérée à ses yeux par Kant), puis en revenant dans un second moment sur le sens de la morale que dégage Adorno en redéfinissant les concepts kantiens.

\footnotetext{
${ }^{11}$ Ibid., p. 224. Adorno insiste sur la dimension critique de l'éthique kantienne - une telle dimension serait absente de l'éthique hégélienne même si elle rend davantage compte des phénomènes sociaux (T. W. Adorno, Probleme der Moralphilosophie, op. cit., p. 245 sq).

12 Ibid., p. 235 : «... wenn Sie etwas von moralischer Dialektik erfaren wollen, und das ist schliesslich der Gegenstand unserer Vorlesung... ».

${ }^{13}$ T. W. Adorno, Dialectique négative, op. cit., p. 189. Voir aussi Ibid., p. 232 : «La contradiction entre liberté et déterminisme n'est pas, comme l'auto-compréhension de la critique de la raison le voudrait bien, contradiction entre les positions théoriques du dogmatisme et du scepticisme, mais une contradiction à l'intérieur de l'expérience que, tantôt libres, tantôt non-libres, les sujets ont d'eux-mêmes ».

14 Ibid., p. 168.

15 Ibid., p. 188.

${ }^{16}$ Ibid.

${ }^{17}$ T. W. Adorno, M. Horkheimer, Dialectique de la raison, op. cit., p. 94.

${ }^{18}$ H. Arendt, Eichmann à Jérusalem, in Hannah Arendt. Le totalitarisme. Eichmann, Paris, Quarto Gallimard, 2002, p. 1149-1151. Kant représente une source d'inspiration pour la théorie politique d'Arendt : H. Arendt, Juger. Sur la philosophie politique de Kant, Paris, Seuil, 2003
} 
Lors du passage de la Critique de la raison pure à la Critique de la raison pratique s'effectue le passage d'une perspective de connaissance sur la liberté à une analyse du pouvoir de détermination de la volonté. Idée régulatrice de l'usage spéculatif de la raison pure, la liberté devient une Idée constitutive de son usage pratique, constitutive d'une morale rationnelle positive. D'après Adorno, reconnaître le statut constitutif de la liberté revient à effectuer un saut théorique absolument contestable et ce serait là la source des problèmes de l'éthique kantienne. L'élément révélateur qui amène Kant à poser la liberté de la volonté ou plutôt, suivant son analyse, à prendre acte de son existence est bien connu : il s'agit de l'expérience décisive que tout être doté de raison fait de la voix de la conscience qui impose en toute clarté le devoir sous la forme d'une proposition indéductible, d'une «proposition synthétique a priori ${ }^{19}$ ». Le « fait de la raison » qui désigne, par analogie avec la donation des faits empiriques, la manière dont la loi morale est donnée à la conscience sans appui d' " aucune intuition, ni pure, ni empirique ${ }^{20}$ » et qui permettrait de déduire la liberté à partir de sa ratio cognoscendi (la loi morale) ${ }^{21}$ est critiqué par Adorno. Il considère que la thèse kantienne essaie de fonder la liberté sur une expérience cruciale en contournant la nécessité d'une démonstration. Pour Adorno, une telle expérience n'a pas valeur de preuve (a), pas plus qu'un donné résistant à l'examen de la raison peut être considéré comme un produit de la raison (b).

Nous allons examiner de plus près ces deux points. On notera au préalable qu'ils ont en commun de partir d'une conception empirique du sujet. En rattachant le sujet de la volonté à un sujet empirique sur le motif qu'il est le seul à pouvoir prendre des décisions, à avoir des impulsions ${ }^{22}$, la Dialectique négative introduit un changement conséquent par rapport à Kant qui étudie le sujet de liberté à la fois comme noumène eu égard à la conscience pure de la loi morale et comme phénomène dans sa conscience empirique. Il y aurait lieu de se demander ici si Adorno n'effectue pas une réduction psychologique de l'appréhension du devoir qui, d'emblée, rend improbable une conciliation avec la perspective kantienne recherchant le fondement d'une obligation inconditionnée. Nous ne faisons qu'indiquer cette voie. Afin de poursuivre notre analyse de la critique adornienne de Kant, nous prêterons surtout attention aux arguments de l'auteur de la Dialectique négative.

a) D'un point de vue phénoménologique (le plan des phénomènes devenant ainsi le seul niveau d'analyse valable pour appréhender la volonté), Adorno montre, en premier lieu, qu'aucune introspection ne saurait fonder la liberté. C'est confondre deux registres différents que de le croire. Par définition, l'introspection ne donne pas accès à une expérience de la liberté ni à son absence. Adorno ne dénonce pas simplement l'illusion des consciences individuelles, il signale que les coordonnées de la liberté ne s'épuisent pas dans un rapport mental de soi à soi, qu'elles dépendent également « de quelque chose d'extramental ${ }^{23}$ », du rapport qu'entretiennent les sujets empiriques avec leur environnement et des effets que celuici a sur leur nature intérieure, sur leur corps. Au Kantien qui rejetterait l'absence de distinction entre les dimensions nouménale et phénoménale, Adorno semble rétorquer que cette perspective duale occulte l'importance du contexte historico-social qui influence sans cesse les consciences et empêche de parler d'un concept absolu de liberté. Pour le théoricien critique, la liberté qui consiste dans la possibilité de rejeter une situation d'oppression

\footnotetext{
${ }^{19}$ Kant, Critique de la raison pratique, op. cit., AK V, 56, p. 127.

${ }^{20}$ Ibid., p. 128 (pour les deux citations).

${ }^{21}$ Ibid, AK V, 6, p. 90 note.

${ }^{22}$ T. W. Adorno, Dialectique négative, op. cit., p. 168.

${ }^{23}$ Ibid, p. 176.
} 
déterminée, de se libérer, reste mêlée à la non-liberté, qui est définie par «l'image de la souffrance $^{24} »$ produite par la répression.

b) En deuxième lieu, en affirmant que la loi morale se manifeste par un fait de la raison, Kant introduirait, selon la lecture adornienne, une contradiction dans sa théorie. La nature double et antithétique de la loi morale paraît évidente quoi qu'en dise le philosophe de Koenigsberg: en tant que loi formelle, issue de la logique pure de la raison pratique, la loi morale est « rationnelle »; mais en se manifestant comme un donné, inaccessible à l'analyse logique, elle est également «non rationnelle ${ }^{25} »$. Loin de fonder la liberté, le fait de la raison est donc l'indice que l'antinomie de la liberté demeure. Cette découverte est essentielle pour comprendre le rapport d'Adorno à Kant.

La lecture du théoricien critique dévoile ainsi un non-dit dans la doctrine kantienne de la liberté. Alors même que son discours affirme une liberté positive, Kant maintient, malgré lui, une notion antinomique de liberté dans sa présentation de la loi morale. Cette ambiguïté que découvre Adorno explique pour une part son rapport complexe à la théorie morale kantienne qui contient, d'après lui, bien plus que ce qu'elle n'expose dans ses thèses.

Le problème fondamental de la philosophie morale n'est donc plus seulement la liberté mais l'enchevêtrement de la liberté et de la non-liberté. Adorno soutient fermement cette thèse, qui, au demeurant, reste un problème. Pour l'étudier, il s'écarte de la méthode de clarification fondée sur la logique formelle ${ }^{26}$ en suivant une « dialectique philosophique » qui prend en compte la dimension historique ${ }^{27}$. La dialectique n'a évidemment pas le sens kantien de «logique de l'apparence ${ }^{28}$ » qui signale une ratiocination vide, elle met à nu les contradictions qui sont dans les choses grâce à une autoréflexion qui découvre le nonidentique masqué sous le principe d'identité et elle assume les contradictions inhérentes à la réalité sociale ${ }^{29}$. Ainsi, au lieu d'essayer de supprimer la contradiction, un examen critique de la morale amène à en prendre la mesure jusqu'au bout.

Les écrits adorniens contemporains de la période de rédaction de la Dialectique négative consacrent alors de nombreux passages à l'examen du concept d'autonomie, qui est la clé de voûte de l'édifice pratique kantien, et qui contiendrait en lui l'ensemble des contradictions de la théorie morale en reposant sur la « relation de la loi et de la liberté ${ }^{30} »$. La douzième conférence de Probleme der Moralphilosophie s'y intéresse en particulier ${ }^{31}$. Examinons le passage commençant ainsi : «L'entrelacement de la liberté et de la loi présenté par Kant est à prendre au sérieux ; ce n'est pas une simple idéologie ${ }^{32} »$. Adorno rejoint Kant pour dire qu'en l'absence d'une loi que la volonté se donnerait à elle-même, il n'y a que du déterminisme ; nous sommes sujets aux influences extérieures, à la tutelle des autres. Et de façon remarquable, en analysant la loi morale dans les termes généraux de la légalité, Adorno étend son propos au-delà du champ moral centré sur l'individu. Il trace une analogie entre les niveaux collectif et individuel : l'absence de droit débouche sur l'état anarchique de la guerre

\footnotetext{
24 Ibid.

${ }^{25}$ Ibid.p. 204.

${ }^{26}$ M. Horkheimer, Théorie traditionnelle et théorie critique [1937], trad. C. Maillard et S. Muller, Paris, Gallimard, 1974, p. 43.

${ }^{27}$ T. W. Adorno, Probleme der Moralphilosophie, op. cit., p. $50 \mathrm{sq}$.

${ }^{28}$ Kant, Critique de la raison pure, op. cit., AK III, 81, p. 150.

${ }^{29}$ T. W. Adorno, Dialectique négative, op. cit., p. 315 sqq.; T. W. Adorno, Probleme der Moralphilosophie, op. cit., p. 51. Voir Jürgen Ritsert, Summa Dialectica. Ein Lehrbuch zur Dialektik, Weinheim, Beltz Juventa, 2017, p. 199214.

${ }^{30}$ T. W. Adorno, Probleme der Moralphilosophie, op. cit., p. 57.

${ }^{31}$ Ibid., p. 180-186.

32 Ibid., p. 181.
} 
de chacun contre chacun présenté par Hobbes dans le chapitre treize du Léviathan ; l'absence de principe de réalité, thématisé par Freud, donne lieu à la tyrannie des désirs addictifs ${ }^{33}$.

Cette relation somme toute positive entre la loi morale et la liberté est contre-balancée par un constat entièrement opposé, qui cette fois s'éloigne de Kant et prend des accents freudiens. Par définition, une loi vient canaliser, réguler les énergies individuelles, aussi demeure-t-elle une contrainte - ce que Kant indiquait - et à ce titre, contre ce que soutient Kant cette fois, la loi morale représente une «menace potentielle pour la liberté34 ». Kant aurait sublimé l'obligation au point d'interpréter en un sens toujours positif le rapport entre la forme contraignante du devoir et la liberté (dans le sens d'une répression de l'amour-propre et d'un accomplissement de l'autonomie). La lecture d'Adorno révèle l'unilatéralité de la position kantienne qui n'envisage à aucun moment l'éventualité que la loi morale puisse avoir un effet répressif sur la liberté : "La sphère de la loi même lorsqu'elle sert formellement l'idée de protéger et de garantir la liberté contient la tendance à abolir la liberté ${ }^{35} »$.

Tout en critiquant le regard sélectif de Kant qui résout harmonieusement la tension entre loi et liberté, Adorno, dans ce passage, examine à nouveau la loi morale à l'aune du concept plus général de «loi », ce qui lui permet de tirer des conséquences aussi bien sur le plan individuel que collectif. Lorsque l'on connaît les critiques acerbes formulées par Adorno à l'endroit du libéralisme politique, il paraît clair que sa critique de l'approche kantienne de la loi (morale) s'en prend tout autant à l'État de droit libéral qu'elle révèle la manière dont les consciences psychiques des sujets modernes sont excessivement réprimées par leur surmoi. La référence à l'État de droit libéral propre à la société bourgeoise, que suggère, à nos yeux, cet effet de la loi sur la liberté apporte un éclairage particulier à la lecture adornienne de la loi morale. Nous allons clarifier ce point.

Après avoir rappelé les rapports contraires qu'entretiennent la loi et la liberté et que présuppose le concept d'autonomie, la douzième leçon des Probleme der Moralphilosophie met en exergue la similitude que la loi morale a avec une règle de droit. Pour ce faire, Adorno relie, selon nous, deux conceptions du droit : la lecture classique de la loi comme commandement ${ }^{36}$ et le lexique moderne de la norme qui caractérise les énoncés prescriptifs de la morale aussi bien que les règles de droit. Si le lexique de la norme rend possible une application aussi bien éthique que juridique de la « loi morale » (comme le souhaite Kant), la lecture impérativiste de la loi, qui peut se dire dans les termes du droit naturel (avec Thomas d'Aquin) ou dans une conception juspositiviste (avec John Austin) ${ }^{37}$, fait de la loi morale une expression du droit. Cette dernière lecture dépasse évidemment l'intention de Kant qui est de fonder l'autonomie de l'éthique autant que celle du droit sur le concept de liberté, comme le rappelle l'introduction à la Métaphysique des Mours. Mais force est de constater que la manifestation du devoir-être par un impératif autorise aussi cette dernière lecture subsumant la loi morale sous le concept de droit. Les commentaires adorniens semblent précisément avoir perçu comment une telle lecture ne peut être si vite écartée pour définir la position kantienne. En d'autres termes, tout porte à croire qu'Adorno découvre un fondement juridique au cœur de la théorie morale kantienne.

\footnotetext{
33 S. Freud, «Formulations sur les deux principes du cours des événements psychiques », trad. J. Laplanche, in Névrose, psychose et perversion, Paris, PUF, 1973, p. 135-143.

${ }^{34}$ T. W. Adorno, Probleme der Moralphilosophie, op. cit., p. 181.

35 Ibid.

${ }^{36}$ Voir T. d'Aquin, Somme théologique, La Loi, la 2 ae Pars, Questions 90-97, trad. M.-J. Laversin, Paris, Desclée, 1935, Question 93 et J. Austin, The Province of Jurisprudence Determined, Cambridge, Cambridge University Press, 1995, Lecture 1.

37 Dans une conception de droit naturel, loi morale et droit sont dans une continuité indistincte ; pour un juspositivisme, la loi morale ne serait qu'un nom pour désigner une loi juridique comme une autre, posée par le législateur.
} 
La Dialectique négative rend compte, selon nous, de la juridicisation de la morale kantienne d'une autre manière lorsqu'elle mentionne la « fiction de la liberté positive ${ }^{38}$ ». En qualifiant la liberté positive de «comme si », Adorno semble faire écho à la lecture que fait Hans Vahinger de la philosophie kantienne en la présentant comme une Philosophie du comme si (1911). À la différence près qu'Adorno perçoit les conséquences théoriques (pour la philosophie kantienne) mais aussi psychosociologiques de l'oubli du «comme si »: les individus socialisés se trompent en pensant que ce modèle de liberté positive est un donné. En qualifiant la liberté de " fiction », Adorno conduit à tirer également des conséquences sur les notions de responsabilité et d'imputation: leur fondement est fragilisé, et avec lui, le fondement de la «justice et du châtiment » que la Dialectique négative trouve au cœur des éthiques classiques ${ }^{39}$. Selon le théoricien de l'école de Francfort, toute tentative de résolution de la troisième antinomie qui consiste à affirmer l'existence de la liberté revient à créer l'illusion d'une règle d'autodétermination et à favoriser les conditions idéologiques de la répression collective. En résumé, l'analyse adornienne montre comment la liberté, posée par la régulation sociale et dont la représentation sert à asseoir l'obéissance ${ }^{40}$, n'est qu'une fiction juridique. Il ne nous semble pas exagéré d'affirmer qu'aux yeux d'Adorno la morale positive de Kant (mais non l'ensemble du questionnement moral kantien) est une doctrine du droit.

Récusant la présentation kantienne qui fait de l'impératif catégorique le fondement de deux champs normatifs distincts, Adorno montre ainsi qu'il n'y a pas d'autonomie normative de la morale et du droit ${ }^{41}$, mais que le fondement moral découvert par Kant est un fondement juridique. On ne peut donc plus adhérer selon lui à la distinction de la Métaphysique des Mours selon laquelle le droit régit la liberté sous son aspect extérieur et l'éthique concerne la liberté sous son aspect intérieur. À suivre Adorno, la liberté perçue dans son rapport à la loi morale est une liberté sans subjectivité, sans moi intérieur - et là se loge l'aliénation -, c'est une liberté qui se dit dans les termes de l'extériorité, dans le langage universel du droit instrument de la répression. La liberté de l'agent moral que décrit Kant semble se réduire uniquement à la liberté de sujets soumis au droit, de sujets de droit.

Dans Kants Kritik der reinen Vernunft (1959), Adorno tire la conclusion suivante de l'autonomie comme définition formelle de la liberté, caractéristique de la société bourgeoise : « la liberté formelle [est celle] des sujets juridiques [, elle] fonde en réalité la dépendance de tout vis-à-vis de tout, le caractère coercitif de la société, sa conformité avec le droit ${ }^{42} »$. En présentant une morale abstraite et rationnelle, le formalisme kantien opère donc, suivant Adorno, la transformation de la morale en droit. La Dialectique négative est encore plus explicite sur ce point : le «formalisme de l'éthique kantienne (...) fait valoir la norme générale du droit ${ }^{43} »$. Avec l'idée que la liberté est liberté formelle ou autonomie, Adorno suggère que l'intériorisation des règles sociales de droit, et surtout de leur esprit, devient telle qu'il n'y a plus de place pour un jugement moral. Des consciences « juridicisées » en quelque sorte, qui font un avec la société, ou qui ne présentent plus de «non-identique irréductible ${ }^{44} »$, ont intériorisé les règles de la répression.

\footnotetext{
${ }^{38}$ T. W. Adorno, Dialectique négative, op. cit., p. 182.

${ }^{39}$ Ibid., p. 167. On peut interpréter ainsi le fait que la troisième partie de la DN s'ouvre sur cette question.

40 Ibid., p. 183.

41 Le lexique kantien est l'inverse du nôtre : ce que nous nommons «morale» est désigné par le terme d' «éthique » dans la Métaphysique des mours et «l'éthique », au sens de théorie morale, désigne la « morale » chez Kant (voir Projet de paix perpétuelle, appendice). Voir J.-F. Kervégan, La raison des normes. Essai sur Kant, Paris, Vrin, 2015.

${ }^{42}$ T. W. Adorno, Kants Kritik der reinen Vernunft, Francfort, Suhrkamp, 1995, p. 88. [Traduit par moi.]

${ }^{43}$ T. W. Adorno, Dialectique négative, op. cit., p. 186.

${ }^{44}$ Ibid., p. 189.
} 
En maintenant le dualisme de la liberté et de la non-liberté, Adorno renvoie dos à dos deux lectures contraires : la réduction déterministe qui rabat l'ordre des raisons sur l'ordre des causes et la réduction volontariste ou légaliste qui oublie que l'ordre des causes est mêlé à l'ordre des raisons. Ces deux interprétations facilitent en effet pareillement l'omnipotence de la société sur l'individu. Si la première réduction donne des bases au naturalisme, comme le note Jürgen Habermas dans son article «'Je suis bien moi-même une parcelle de la nature' Adorno sur l'intrication de la nature et de la raison. Réflexions sur le rapport entre la liberté et ce dont il est impossible de disposer ${ }^{45}$ ", la seconde, que nous avons mise à nu ici, soutient une juridicisation des rapports sociaux qui, en faisant croire que les lois ont un caractère spontané, est répressive.

II.

Les critiques d'Adorno déconstruisent l'édifice pratique kantien, et à travers lui toute théorie morale, puisque la «philosophie morale kantienne est la philosophie morale par excellence », pour reprendre le mot de Probleme der Moralphilosophie. Cette conclusion est déconcertante. Il y a lieu de se demander si une philosophie morale, quelle qu'elle soit, est encore possible. Afin de répondre à cette interrogation, il n'est pas inutile de noter que l'examen métacritique de la raison pratique, s'il ne débouche pas sur une reformulation de l'éthique kantienne par d'autres moyens, adopte cependant l'économie générale de celle-ci en attribuant aux motifs centraux de Kant une fonction épistémologique analogue tout en les redéfinissant : c'est le cas des notions de liberté, de volonté et de spontanéité ainsi que de l'impératif catégorique. Ces concepts ne sont pas vidés de leur substance, ils sont redéfinis dans une configuration qui conserve leur fonction pour la compréhension de la morale. Nous allons, dans un second moment, regarder de plus près chacune de ces notions et les changements opérés par Adorno les concernant.

À propos du concept de liberté, on peut se demander si l'idée d'une dialectique de la liberté se manifestant différemment à travers l'histoire apporte un élément nouveau au problème de l'obligation morale ressentie par des sujets individuels. Indépendamment de l'examen métaphysique, l'idée que la liberté et le déterminisme ne sont pas des options alternatives définitives au niveau individuel mais que leur opposition se rejoue constamment dans le conflit entre loi morale et présomption de l'amour de soi $^{46}$ est présente chez Kant. Mais si l'issue du conflit est incertaine pour cet auteur, la voie à suivre reste claire. C'est là un point de divergence avec la position adornienne. En effet, l'insistance sur la dynamique dialectique qui fait émerger des éléments de liberté dans un état de non-liberté rend compte de la confusion qui entoure les situations morales ; non pas que ces dernières offrent forcément une «complexité » épistémique ${ }^{47}$, mais parce que l'incertitude concernant la pseudo-volonté libre n'est pas dissipée une fois pour toutes.

Toutefois, même s'il la déplore pour ses effets répressifs, Adorno tient pour vrai l'idée kantienne selon laquelle il n'y a de responsabilité qu'à condition de postuler une liberté. Sans le postulat de la liberté, il n'y a aucune base permettant de réprouver les crimes d'Auschwitz ou d'empêcher qu'une telle horreur survienne à nouveau ${ }^{48}$. En même temps, et c'est toute la difficulté saisie par Adorno, il n'y a pas de place pour une liberté individuelle éthique dans

\footnotetext{
45 J. Habermas, Entre naturalisme et religion. Les défis de la démocratie, trad. C. Bouchindhomme et A. Dupeyrix, Paris, Gallimard, 2008, p. 126 sqq.

${ }^{46}$ Kant, Critique de la raison pratique, op. cit., 1. I, chap. 3.

47 A. Wellmer, Ethik und Dialog. Elemente des moralischen Urteils bei Kant und in der Diskursethik, Francfort, Suhrkamp, 1986.

${ }^{48}$ T. W. Adorno, Zur Lehre von der Geschichte und von der Freiheit, Francfort, Suhrkamp, 2001, p. 280 sq.
} 
une société répressive. Dans des conditions sociales ne laissant pas de place au non-identique, «la non-liberté a préséance sur la libertét9 ».

L'aporie mise au jour par Adorno qui entache la question de la liberté - on ne peut ni postuler de liberté dans un régime autoritaire, ni envisager sa disparition complète - empêche en toute rigueur de parler de situation morale. L'auteur de la Dialectique négative semble nous dire qu'on aurait beau jeu de mettre en évidence les coordonnées d'une situation morale une fois modifié le contexte social et en toute ignorance des termes dans lesquels elle s'est présentée aux consciences. N'acceptant pas sans doute toutes les conséquences morales relativistes de cette vue auxquelles il est difficile de répondre et qui sont très problématiques étant donné l'horreur absolue des camps d'extermination, Zur Lehre von der Geschichte und von der Freiheit suggère qu'il reste possible de parler d'obligation morale si l'on envisage l'existence de manifestations, plus ou moins imparfaites, plus ou moins étouffées, de liberté: «Si Auschwitz a pu arriver en premier lieu, c'est probablement parce qu'aucune liberté véritable n'existait encore ${ }^{50} »$. Il paraît possible d'envisager un continuum de degrés de liberté à travers l'histoire, en considérant que des éléments de liberté sont toujours aux prises avec une non-liberté. L'approche de Kant est donc modifiée dans la lecture adornienne par la psychanalyse freudienne - la liberté est plus ou moins réprimée - et par une philosophie de l'histoire sans idée de progrès - la liberté n'est pas une propriété individuelle mais dépend d'un contexte socio-historique.

En un sens, il ne reste pas grand-chose de la conception kantienne après son examen «métacritique »; le postulat de la liberté devient l'exigence inaccessible d'une éthique formelle et individualiste, seules des traces de liberté sont repérables à la marge. La liberté n'est perceptible que comme «négation déterminée ${ }^{51}$ » des formes sociales qui empêchent les conditions mêmes de son apparition. Ou encore, note la Dialectique négative, "la liberté devient concrète à partir des formes changeantes de la répression : en s'opposant à elles ${ }^{52}$ ». Alors que l'autonomie kantienne semble le résultat d'un moi fort qui sort vainqueur de toute influence empirique ${ }^{53}$, les formes de liberté se manifestant par une négation déterminée restent prises dans un processus conflictuel : un acte libre est une percée provisoire et peut-être illusoire hors de la non-liberté, puisque l'individualité qu'il exprime est elle-même un « moment de la société marchande ${ }^{54} »$.

La perception de formes de liberté dues à une négation déterminée suppose de revoir le mode de détermination de la volonté. L'alternative (kantienne) non contradictoire entre une volonté pure déterminée par la raison et une volonté hétéronome déterminée par des intérêts empiriques rate, selon Adorno, le principe même de la volonté qui, "force de la conscience ${ }^{55} »$, peut transformer cette dernière en résistance. L'incongruité du partage strict consiste à faire de la conscience pure le lieu de la volonté sans prendre en compte le fait qu'à travers l'imagination celle-ci est au contact du corps. La dimension psychosomatique des sujets empiriques oblige à ne plus abstraire la volonté des impulsions corporelles et des énergies pulsionnelles. En isolant la volonté de son environnement social et de la médiation du corps, en la caractérisant par la logique d'une raison (pratique) pure, Kant et, avec lui, toute la conception rationaliste depuis Descartes ratent, suivant Adorno, la manière dont la volonté est engagée dans une dialectique opposant le mental et l'extra-mental.

\footnotetext{
${ }^{49}$ T. W. Adorno, Dialectique négative, op. cit., p. 189.

${ }^{50}$ Adorno, Zur Lehre von der Geschichte und von der Freiheit, op. cit., p. 279. [Traduit par moi.]

${ }^{51}$ T. W. Adorno, Dialectique négative, op. cit., p. 182.

${ }^{52}$ Ibid., p. 207.

${ }^{53}$ Kant, Critique de la raison pratique, op. cit., 1. I, ch. 1, § 8, AK V, 33, p. 130 sq.

${ }^{54}$ T. W. Adorno, Dialectique négative, op. cit., p. 206.

${ }^{55}$ Ibid., p. 190.
} 
Sans cette représentation d'une «détermination dialectique de la volonté ${ }^{6}$ », l'indice même de la liberté comme épiphénomène ou rupture de l'enchaînement causal, à savoir la spontanéité, devient pur mécanisme. En attribuant la spontanéité à la conscience pure d'un sujet suivant les lois de la raison, Kant commet l'erreur de réintroduire subrepticement la causalité qu'il avait rejetée comme expression du déterminisme, dans le rapport entre raison et volonté : en tant que loi, la loi morale relève du registre de la causalité. Ainsi la thèse radicale d'une « liberté positive » issue d'une autodétermination de la volonté donne raison à la thèse opposée du déterminisme : la volonté est déterminée par la raison pratique pure dont la logique, si l'on suit à la lettre l'idée kantienne de l'unité de la raison pure ${ }^{57}$, relève de la causalité. La volonté pure se révèle être l'intériorisation de la répression externe.

Comment sortir de cette impasse ? Paradoxalement, Adorno trouve une issue en considérant la volonté comme la faculté d'un sujet empirique. Alors que le risque de déterminisme paraît accru pour une telle volonté, Adorno y répond en réinvestissant la notion de spontanéité.

Davantage que Kant même qui déporte la preuve de la liberté sur la loi morale, Adorno attribue un rôle clé à la spontanéité en tant qu'indice de liberté. L'explication de ce « saut » dans la chaîne causale des événements ne fait plus appel au caractère intelligible des êtres humains mais à une autre conception de la conscience : la conscience psychologique d'un moi dont les énergies pulsionnelles expriment aussi des expériences psychosomatiques. La logique pulsionnelle, réactive, explique l'impulsion dont la volonté n'est pas à l'initiative, mais qu'elle accompagne. Sans la main qui tressaille, « sans l'aspect moteur de la réaction ${ }^{58}$ », il n'y a plus de volonté, note Adorno : la question n'est pas que la volonté contrôle le corps ou soit principe actif, comme le conçoit le schéma rationaliste dominant la pensée occidentale, mais que les sujets soient appelés à agir, et soient disposés à suivre une inclination qui ne pourrait pas naître du seul univers rationnel, lequel est perméable à la répression (puisque la loi morale a des similitudes avec le droit) et au déterminisme (car la loi de la liberté reste un cas d'espèce de la causalité) - ces deux concepts rendant compte en bout de chaîne du même phénomène social. La spontanéité qui correspond à une «expérience vague ${ }^{59}$ » échappe à l'analyse rationnelle en étant fortuite et en exprimant une certaine liaison entre la raison et le corps. C'est là l' «aspect fuyant »du « supplément »(das Hinzutretende), caractéristique qui n'est pas problématique puisqu'à la différence du caractère intelligible de Kant, celui-là ne répond pas à la logique de la raison.

Le rapport de la spontanéité à l'action morale fait appel à un troisième terme, signe sans doute que l'approche de la morale resterait subjective et arbitraire sans médiation objective. Il semble que la mention d'un «nouvel impératif catégorique » ait pour fonction d'exhiber un repère objectif. Ce qu'est exactement cet impératif pour Adorno est difficile à saisir, car il échappe à une conceptualisation complète, mais il exprime sans ambiguïté une nouvelle approche de la morale. L'erreur consisterait à interpréter le nouvel impératif catégorique uniquement avec des catégories kantiennes, même si celles-ci peuvent aider à se repérer dans un premier temps. Inversement, il n'est pas envisageable de voir dans l'impératif adornien une simple parodie de l'impératif kantien; son contenu ne saurait en effet être pris à la légère : «penser et agir en sorte que Auschwitz ne se répète pas, que rien de semblable n'arrive ${ }^{60} \gg$.

De façon très intuitive, cette formule exprime le devoir inconditionné d'un « jamais plus » valable pour toute l'humanité et à ce titre est bien catégorique et porteuse d'une

\footnotetext{
${ }^{56}$ Ibid.

${ }^{57}$ Kant, Critique de la raison pratique, op. cit., AK V, 121, p. 245.

${ }^{58}$ T. W. Adorno, Dialectique négative, op. cit., p. 181.

${ }^{59}$ Ibid., p. 179.

${ }^{60}$ Ibid., p. 286.
} 
universalité. La difficulté à faire de l'impératif adornien un principe universel demeure néanmoins et c'est sans doute un aspect recherché par le théoricien critique, soupçonnant tout universel de n'être qu'illusion. L'un des points soutenant cette difficulté vient de ce qu'à l'opposé de l'impératif kantien, formel et rationnel, l'impératif adornien est substantiel et se

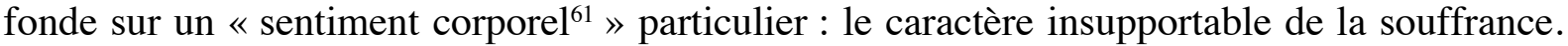
On est renvoyé tacitement à la «bonne mimésis » que recèle la rationalité esthétique thématisée dans la Théorie esthétique ${ }^{62}$ : rompant avec la pensée conceptuelle et avec le mimétisme comportemental, une sensibilité prenant sa source dans l'empathie et réconciliée avec la raison découvre autrui (ou un objet) dans sa différence même ${ }^{63}$ et ouvre sur une voie inédite pour répondre à sa présence. À la manière des romans psychologiques ou des récits autobiographiques qui convoquent des traits humains communs en parlant du plus intime de soi - si l'on nous passe cette comparaison -, l'élément le plus subjectif qu'est la douleur revêt un caractère général, justifiant le mouvement qui disposerait chacun à réagir face à son spectacle sans que cette réaction soit garantie. Le sentiment physique suscité par l'exposition de la souffrance est le «moment de surgissement de la moralité64 » au sens où un sujet prend confusément conscience de quelque chose à travers son corps agi qui réagit.

De la même manière que le « fait de la raison » était indémontrable et faisait du « « Tu ne dois pas » un principe métaphysique, un principe qui vise au-delà de la pure facticité », le nouvel impératif catégorique en appelle à une forme d'absolu pour les sujets empiriques dont la réalité est corporelle. En ce sens, « la métaphysique a glissé dans l'existence matérielle ${ }^{65}$ » explique Métaphysique. Concept et problèmes. Adorno retient de Kant l'idée que le fondement moral n'est pas déductible ${ }^{66}$, ou, pour le dire avec Jean-François Lyotard, que «l'autorité ne se déduit $\operatorname{pas}^{67}$ ». Si l'on accepte la possibilité d'une mimésis empathique conciliée à la raison, le caractère indémontrable de la morale pose moins de problèmes à l'approche adornienne qu'au cadre kantien : la morale n'étant plus un produit de la raison, mais issue d'un sentiment corporel - on lit même « fondée $\operatorname{par}^{68}{ }^{\text {»-}}$, l'aspect indéductible de la morale ne met pas la raison en porte-à-faux avec elle-même.

L'impératif matériel «extralogique ${ }^{69}$ » est le fait du corps, comme l'impératif formel est le fait de la raison. Sans doute est-il impossible de démontrer que l'impératif matériel a les caractéristiques requises pour fonder une morale universelle (ce qui rend son statut contestable pour un Kantien), mais il est tout aussi difficile de nier l'évidence du devoir qu'il impose, à savoir l'attention minimale que requiert tout être corporel du fait de sa sensibilité. La difficulté qu'il y a à exprimer dans un langage conceptuel l'impératif adornien n'est pas non plus un défaut; elle correspond à l'esprit même de la dialectique négative qui s'est donnée pour tâche de «mettre à jour le non-conceptuel au moyen de concepts sans l'assimiler à eux ${ }^{70} \gg$.

En ce qui concerne la question de la détermination de la volonté, l'impératif catégorique et l'impératif extralogique n'apportent pas plus l'un que l'autre la garantie qu'une

\footnotetext{
${ }^{61}$ T. W. Adorno, Métaphysique. Concept et problèmes, trad. C. David, Paris, Payot, 2006, p. 173.

${ }^{62}$ T. W. Adorno, Ästhetische Theorie, Francfort, Suhrkamp, p. 326. Voir J. Früchtl, Mimesis. Konstellation eines Zentralbegriffs, Königshausen \& Neumann, 1986 et Jürgen Ritsert, op. cit., p. 204-207.

${ }^{63}$ A. Honneth, « The Other of Justice », trad. John Farrell, in S. K. White (dir.), The Cambridge Companion to Habermas , Cambridge, Cambridge University Press, 1995, p. 289-323, ici : p. 299.

${ }^{64}$ T. W. Adorno, Dialectique négative, op. cit., p. 286.

65 Ibid.

${ }^{66}$ F. Freyenhagen, Adorno's Practical Philosophy, Cambridge, Cambridge University Press, 2013, p. 117.

${ }^{67}$ J.-F. Lyotard, Le différend, Paris, éd. de Minuit, 1983, § 203-204.

${ }^{68}$ T. W. Adorno, Métaphysique. Concept et problèmes, op. cit., p. 173.

${ }^{69}$ Ibid.

${ }^{70}$ T. W. Adorno, Dialectique négative, op. cit., p. 16.
} 
action sera motivée par eux ; une différence est à noter toutefois : l'impuissance de la volonté chez Kant concerne sa faiblesse à pouvoir motiver l'action, tandis que pour Adorno l'impuissance de la volonté questionne sa structure même. Le test d'universalisation de la «typique de la faculté de juger pratique » apporte une justification aux normes morales en supposant la totalité harmonieuse du royaume des fins, le nouvel impératif catégorique se comprend par opposition à un « faux collectif ${ }^{71} »$.

En suivant l'économie générale de l'éthique kantienne, la conception adornienne répond en tout point à celle-ci, et à travers elle à toute philosophie morale. Contre toute morale positive, Adorno révèle bien l'absence de sécurité qui caractérise la morale ${ }^{72}$ : la réponse de la volonté mue par l'impulsion corporelle fait état d'un rapport tragique au monde en ce que rien n'assure qu'elle soit le fruit d'un acte spontané libéré de la réification totale. Kant expose les conditions de possibilité de la morale dans un monde non totalement emprunt de barbarie ; Adorno se demande si une action libre est encore possible lorsque la culture a échoué. Les traces de la liberté sont repérées dans des expressions du refoulé, dans «le souvenir de ce qui a été éliminé73 ${ }^{7}$; et la question demeure ouverte de savoir si, à l'occasion même de leur manifestation, ces formes de liberté ne seront pas l'expression d'une nouvelle barbarie - ce qui traduit sur le plan moral la chute de la métaphysique ${ }^{74}$.

Les faiblesses de la théorie morale kantienne sont occultées ou minimisées par le cadre d'une éthique volontariste : le présupposé de la liberté, l'incertitude cognitive concernant la motivation d'une action, et plus généralement l'incapacité à dire s'il y aura jamais une action morale en ce monde ${ }^{75}$. Tout en exhibant ces points laissés en suspens, Adorno révèle le caractère aporétique de la morale même, en demandant si morale il peut y avoir, si même cette possibilité envisagée par Kant n'est pas pure spéculation. L'envers de la conception adornienne est, semble-t-il, de pouvoir excuser l'inaction.

En conclusion, malgré les critiques qu'il adresse à la conception kantienne de la morale, Adorno retient d'elle son apport épistémologique. Elle a su clarifier les coordonnées du problème moral en découvrant l'antinomie de la liberté ${ }^{76}$. En repensant la morale après Auschwitz, Adorno ne se contente pas de montrer l'insuffisance de la liberté positive postulée par Kant, qui transforme la morale en droit et facilite l'intériorisation de la répression. S'en prenant à toute morale positive, la Dialectique négative révèle la fragilité inhérente à la morale : sentiment corporel éveillant la volonté et dialectique de la liberté ne donnent des indices que de ce qui ne doit pas être, mais non de ce qui doit être. Par définition, comme l'examen métacritique l'a montré, la morale reste liée à une critique dénonciatrice dont la qualité incontestable réside dans le fait qu'elle offre des éléments pour identifier le non-sens ${ }^{77}$.

\footnotetext{
${ }^{71}$ Ibid., p. 221.

${ }^{72}$ Ibid., p. 190.

${ }^{73}$ Ibid., p. 181.

${ }^{74}$ Ibid., p. 317.

${ }^{75}$ Kant, Théorie et pratique, trad. F. Proust, Paris, GF, AK VIII, 284, p. 56.

${ }^{76}$ T. W. Adorno, Métaphysique. Concepts et problèmes, op. cit., 15 $15^{\mathrm{e}}$ leçon; voir A. Wellmer, «Solidarity with Metaphysics after its Fall (1988), in Endgames : The Irreconcilable Nature of Modernity. Essays and Lectures, trad. D. Migley, Cambridge, The MIT Press, 1998, p. 183-202, ici : p. 184.

${ }^{77}$ Cette expression d'Albrecht Wellmer (Ethik und Dialog, op. cit., p. 127) prend un sens élargi ici. Il parle quant à lui d'une éthique « éliminant le non-sens » et y voit là la tâche d'une éthique faillibiliste de la discussion.
} 